\title{
Expert Consensus on Some Issues of Cerebral and Carotid Vascular Ultrasonography
}

\author{
The Professional Committee of Vascular Ultrasound of Stroke Prevention and Treatment Expert, Committee of \\ the National Health Commission; The Professional Committee of Superficial Organ and Peripheral Vascular \\ Ultrasound of the Chinese Medical Ultrasound Engineering; The Professional Committee of Craniocerebral and \\ Cervical Vascular Ultrasound of the Chinese Medical Ultrasound Engineering
}

Received July 11,2020; revision received August 1, 2020; accepted August 8, 2020

\begin{abstract}
The consensus was established based on some questions of cerebral and carotid ultrasound examination in practice through the collection, elucidation and explanation by three professional committees in the field of vascular ultrasonography, including the Professional Committee of Vascular Ultrasound of Stroke Prevention and Treatment Expert, Committee of the National Health Commission; the Professional Committee of Superficial Organ and Peripheral Vascular Ultrasound of the Chinese Medical Ultrasound Engineering; the Professional Committee of Craniocerebral and Cervical Vascular Ultrasound of the Chinese Medical Ultrasound Engineering, in order to promote the professional field and the level of technology standardization. It has important value in clinic leading and professional guidance.
\end{abstract}

Key words: Stroke; Carotid arteries; Ultrasonography; Cerebral vascular; Expert consensus

Advanced Ultrasound in Diagnosis and Therapy 2021;02:153-162

DOI: 10.37015/AUDT.2021.200057

$\mathrm{W}$ ith the development of stroke prevention and treatment projects by the National Health Committee, combined assessment of carotid and intracranial arteries by vascular ultrasound has become an important screening modality in populations at high risk of stroke. However, diagnosis using intracranial and carotid ultrasound still has several problems that remain to be fully explained. In the present consensus, we collected 3658 valid questionnaires from doctors of hospitals at all levels across 34 provinces and autonomous regions. To improve diagnosis, treatment standards, and technical expertise using intracranial and carotid ultrasound, we determined some common problems of carotid ultrasound according to experts in the field.

\section{Value of B-mode, CDFI, and Pulsed Wave Doppler in the Diagnosis of Carotid Stenosis \\ Expert opinion: Conventional carotid ultrasound}

comprises B-mode (brightness mode), color Doppler flow imaging (CDFI), power Doppler imaging, and pulsed wave Doppler to detect hemodynamic parameters. During detection, the Doppler angle between blood flow and sound beam should be kept less than $60^{\circ}[1,2]$.

\subsection{B-mode}

B-mode (brightness mode) is mainly used to evaluate the structure and intima-media thickness (IMT) of the carotid artery, plaque size, morphology, and acoustic (echo) characteristics, as well as to calculate diameter stenosis rate and area stenosis rate. Clinicians can then make a preliminary judgment about the stenosis degree and etiological characteristics of affected vessels.

\section{$1.2 \quad C D F I$}

CDFI was used to observe blood filling, direction, velocity distribution of blood flow, and localization of responsible vessels. It can further improve the contrast of

\footnotetext{
* Corresponding authors: Yang Hua, Department of Vascular Ultrasonography, Xuanwu Hospital, Capital Medical University, Beijing. e-mail: dryanghua99@163.com; Tianan Jiang, Department of Ultrasonography, the First Affiliated Hospital, Zhejiang University School of Medicine, Hangzhou. e-mail: tiananjiang@zju.edu.cn; Chaoyang Wen, Department of Ultrasound, Peking University International Hospital, Beijing.e-mail: wency301@vip.sina.com.
} unrestricted use, distribution and reproduction in any medium provided that the original work is properly attributed. 
hypoechoic or echolucent plaques, as well as increase the detection rate of ulcer plaques.

\subsection{Power Doppler Imaging}

Power Doppler imaging can improve the sensitivity of low-velocity blood flow detection in arteries with severe stenosis or near occlusion.

\subsection{Pulsed Wave Doppler}

Pulsed wave Doppler was used to detect gradient changes in blood flow velocity caused by stenosis, including peak systolic velocity (PSV) and end diastolic velocity (EDV) in stenosis, prestenotic segment, and poststenotic segment. On this basis, to improve the accuracy with which the degree of carotid stenosis was evaluated, the velocity ratio between the stenosis and the prestenotic segment was calculated, as was the velocity ratio between the stenosis and the poststenotic segment.

\section{Comprehensive Use of Linear Array, Convex} Array, and Micro-convex Probe to Improve the Accuracy of Velocity Measurement in the

\section{Carotid Artery}

Expert opinion: To improve the accuracy of carotid artery velocity detection, clinicians must focus on two areas: (1) ensuring that B-mode and CDFI of the carotid artery confer clear images; (2) a sample like $\leqslant 60^{\circ}$ angle between the blood flow and the sound beam. Each of the three probes has its own advantages, and all of them should be used in combination. To examine the common carotid artery, which has a superficial position, clinicians routinely use a high-frequency linear array probe (Fig. 1A) with very high two-dimensional imaging resolution and clear CDFI. However, there are limitations regarding the detection of lesions in the middle and distal segments of the internal carotid artery, as well as in the initial segments of the vertebral and the subclavian arteries. In patients with a high bifurcation position, a short and thick neck, or obesity, a convex array probe (Fig. 1B) or micro-convex probe (Fig. 1C) can be used instead. The convex array probe increases the length of the internal carotid artery that can be displayed, improves the resolution of middle and distal segment lesions, and improves the accuracy of flow velocity measurement in the deep and distal segment arteries [1]. The microconvex array probe confers significantly better image resolution than the convex array probe. It is therefore suitable for the detection of deep and distal segment arteries, especially in patients with short and thick necks and obese patients. To improve the accuracy of examination, the combination of these three probes is recommended.
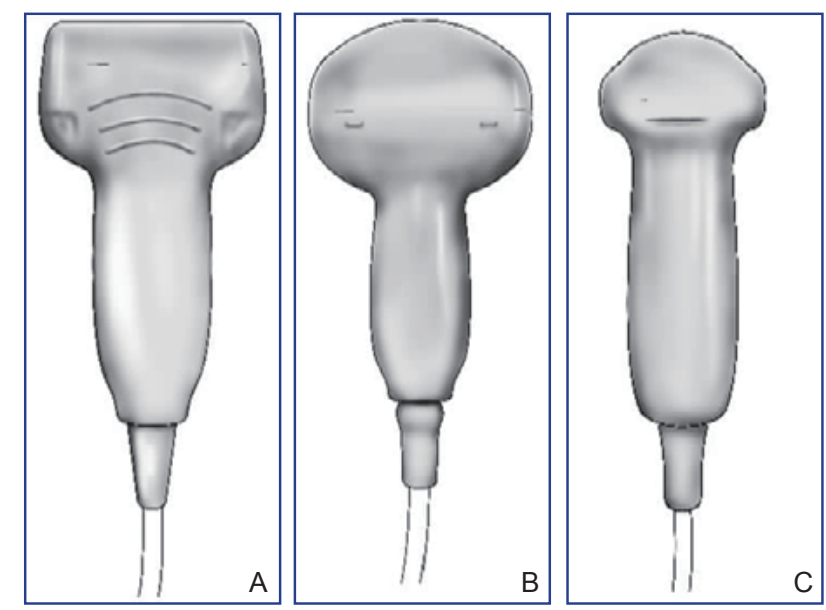

Figure 1 Probes for carotid artery ultrasonography. A, B, and C are the linear array probe, convex array probe, and micro-convex probe, respectively.

\section{Definition of the Proximal, Middle, and}

\section{Distal Segments of the Common Carotid Artery}

Expert opinion: The right common carotid artery originates from the innominate artery, while the left common carotid artery originates from the aortic arch. To describe the location of common carotid artery lesions more accurately, the artery should be divided into proximal, middle, and distal segments, with the upper and lower poles of the normal thyroid as markers. The proximal part is the common carotid artery from the branch of the aortic arch (left) or innominate artery (right) to the lower pole of the thyroid. The middle part is between the upper and lower pole of the thyroid, while the distal part is from the upper pole of the thyroid to near the bifurcation of the artery (Fig. 2).

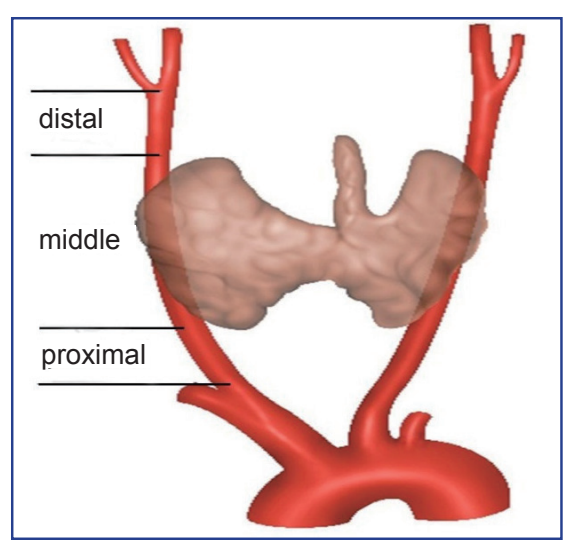

Figure 2 Diagram of the common carotid artery segments.

\section{Anatomical Location of the Carotid Bulb,} Sinus, Bifurcation, and Proximal Segment of the Internal Carotid Artery

Expert opinion: The location landmark is the 
bifurcation point of the common carotid artery, which is divided into the internal and external carotid arteries (Fig. 3).

\subsection{Carotid bulb and proximal internal carotid artery}

The bulb of the carotid artery is a local widening at the start of the internal carotid artery, which is distal to the common carotid artery bifurcation. The proximal internal carotid artery refers to the part that is farthest from the bulb and lies parallel to the anterior and posterior walls of the internal carotid artery.

\subsection{Carotid sinus}

A carotid sinus is a pathophysiological term used to describe an area of high pressure and specific chemoreceptors. Ultrasound cannot determine its location, and it is generally distributed within the adventitia of the carotid artery between the bifurcation and the bulb.

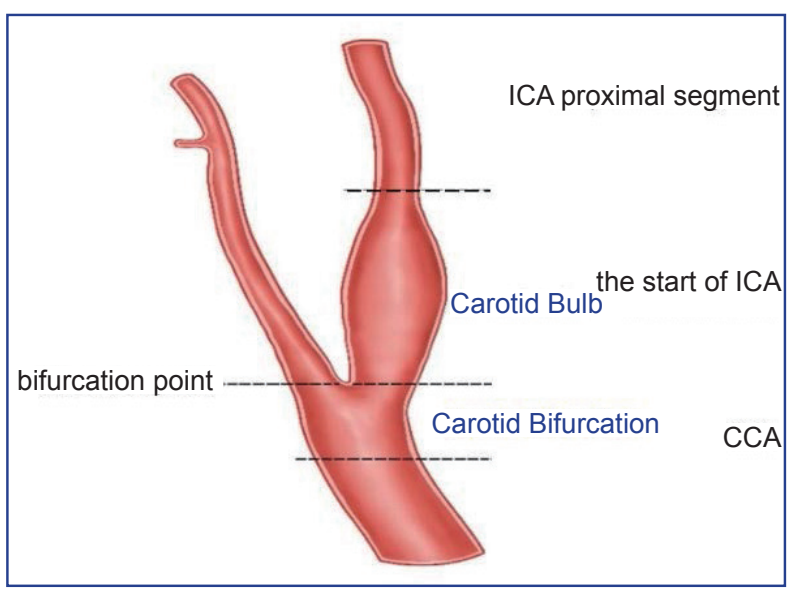

Figure 3 Diagram of common carotid artery bifurcation, carotid bulb, and proximal segment of the internal carotid artery.

\subsection{Carotid bifurcation}

The dilated part of the artery from the horizontal segment of the distal bifurcation to the proximal (initial) segment of the internal carotid artery.

\section{Evaluating the Accuracy of Carotid Ultrasound}

\section{Reports of "Arteriosclerosis"}

Expert opinion: Carotid ultrasound reports of "arteriosclerosis" are not generally accurate. Arteriosclerosis is a general term for a kind of disease characterized by thickening, stiffness, and loss of elasticity in the arterial wall. It can be further categorized as atherosclerosis, calcification of the artery media, and arteriosclerosis [3]. It follows that atherosclerosis is a specific type of arteriosclerosis.

Carotid atherosclerosis is a chronic and progressive inflammatory change in the carotid artery; it is characterized by progressive thickening and structural disorder of the intima-media [4]. The earliest manifestation of atherosclerosis is thickening of the intima-media. Atherosclerotic plaques then form, which may develop into stenosis or occlusion with disease progression. According to the vascular diameter and hemodynamic parameters, the degree of carotid stenosis can be comprehensively evaluated and divided into four grades: $<50 \%, 50 \%-69 \%, 70 \%-99 \%$ stenosis, and occlusion [1].

Ultrasonic diagnosis of carotid atherosclerosis should follow the basic principles of location, quality, and quantity. The ultrasonic results should include the following measurements: (1) Location, including the site and artery involved in the lesion; (2) Qualitative observations, namely uneven thickening of the IMT, the characteristics of the plaque(s) (single or multiple), and the presence of atherosclerotic lesions; (3) Quantitative measurements, namely the degree of stenosis and occlusion (complete occlusion and near occlusion). The ultrasonic report of atherosclerotic lesions may indicate uneven thickening of the IMT, with single or multiple plaque(s) formation. If stenosis has occurred, the results should clarify the artery involved and the degree of stenosis. For example, "stenosis of the right internal carotid artery (proximal segment: 50\%-69\%)".

\section{Standardized Measurement and Description of IMT and Plaque Size}

Expert opinion: The measurement of carotid IMT and plaques is the basis of carotid atherosclerosis evaluation.

\subsection{Ultrasonic measurement of IMT}

In the B-mode, the IMT of the far wall was measured. In this case, the measurement requirements are as follows: the probe must be parallel to the vessel wall; the sound beam should be perpendicular to the vessel wall; the longitudinal section should be combined with the transverse section; and the IMT should be measured in the far wall of artery $(1.0-1.5 \mathrm{~cm}$ below the bifurcation level) in a region free of plaque. The vertical distance from the lumen-intima to media-adventitia interfaces is measured to provide the combined thickness of the intima and media of the vascular wall [4]. It is considered normal below $1.0 \mathrm{~mm}$, and increased between 1.0 and 1.4 $\mathrm{mm}$. For values equal to $1.5 \mathrm{~mm}$ or higher, the IMT turns into a "plaque" [4].

\subsection{Atherosclerotic plaque}

A plaque is a focal structure encroaching into the artery lumen by at least $0.5 \mathrm{~mm}$ or $50 \%$ of the surrounding IMT value [5] or demonstrating IMT 
thickness greater than $1.5 \mathrm{~mm}$. The measurement and description of plaques should focus on their location, size, shape, and echo.

6.2.1 Plaque location: The B-mode can be obtained by combining the transverse and longitudinal sections (antero-posterior, external-internal), and dynamic adjustment should be used to comprehensively determine the location of plaque. For semi-circular or circular plaque distributions, the extent of plaque distribution on the vessel wall should be determined and described based mainly on the transverse section [1].

6.2.2 Plaque size: expressed in length $(\mathrm{mm}) \times$ thickness $(\mathrm{mm})$. The length of the plaque protruding from the lumen is the horizontal distance between its upper and lower ends, and its thickness is the vertical distance from the highest point (top) of the plaque surface to the upper edge of the adventitia of the vessel wall.

6.2.3 Plaque shape: categorized as regular, irregular, or ulcerated (when the fiber cap on the surface of plaque is broken and discontinuous, forming the "volcanic crater" sign, the length and depth of the crater are both $\geqslant 2.0$ $\mathrm{mm}$, and CDFI shows blood flowing into the plaque).

6.2.4 Plaque echo: categorized as homogeneous and heterogeneous echo. Homogeneous echo can be further divided into homogeneous hypoecho, homogeneous isoecho, and homogeneous hyperecho. When the areas of the echoes differ by $\geqslant 20 \%$, heterogeneous echo is identified. Researchers and clinicians should always note which the main echo is.

\section{Description of Plaque Location}

Expert opinion: The plaque can be located by combining the scans of the longitudinal and transverse sections.

\subsection{Longitudinal section}

With regards to anatomical position, a plaque can be located in the proximal, middle, or distal common carotid artery, bifurcation, carotid bulb, proximal segment of the internal carotid artery, or the external carotid arteries. If the plaque is long, it can be described in terms of "extending to," such as "extending from the bifurcation of the common carotid artery to the bulb."

\subsection{Transverse section}

The largest cross-section of the plaque can be defined according to the "eight points method" for positioning and classification. Those points are the front wall (ventral side), the rear wall, the inner wall (trachea side), the outer wall, the front inner wall, the rear inner wall, the front outer wall, and the rear outer wall [6].

\subsection{Plaque description example}

(1) At the bifurcation of the right common carotid artery, $16.5 \mathrm{~mm} \times 2.3 \mathrm{~mm}$ isoechoic flat plaques can be detected on the rear inner wall; (2) in the left common carotid artery, $25.0 \mathrm{~mm} \times 3.3 \mathrm{~mm}$ heterogeneous, irregular plaques with mainly hyperecho and acoustic shadow can be detected on the front inner wall from the bifurcation extending to the bulb. These are related to calcified plaques [5].

\section{The Definition of Vulnerable Plaque and the Clinical Significance of Ultrasonography}

Expert opinion: The vulnerability of a plaque can be judged using comprehensive analysis of plaque morphology, echo, fibrous cap integrity, and other information.

Vulnerable plaques refer to plaques with a tendency to rupture, prone to thrombosis, and/or rapidly developing into responsible lesions on the basis of carotid atherosclerosis [7], which are closely related to ischemic cerebrovascular disease. The main characteristics of vulnerable plaques identified by ultrasound include plaque rupture (ulcer plaque), plaque hemorrhage or large lipid core (more than $40 \%$ of the plaque area), and neovascularization.

\subsection{Plaque rupture}

In ruptured plaques, the fibrous cap on the surface is broken and pulsating with the heart beat. The broken fibrous cap can be seen in the lumen, a thrombus may have formed on the surface, or ulceration may have occurred. Ultrasonic features of ulcerative plaque: the fibrous cap on the surface of the plaque is broken and discontinuous, forming a "volcanic crater" sign,

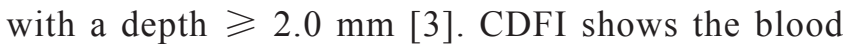
flow entering the plaque. The surface of an ulcerative plaque can become the site of platelet aggregation and thrombosis, and it may fall off, leading to arterial embolism and stroke [8].

\subsection{Lipid core with hemorrhaging in the plaque}

Plaques show hypoechoic or echolucent characteristics, and hemorrhaging or large lipid core is related to plaque progression. Hemorrhage in the plaque can occur after rupture of the fibrous cap; if there is secondary platelet aggregation, a thrombus may form. Hemorrhage can also occur after the rupture of neovascularization at the base of the plaque. The larger the hemorrhage or lipid core, the higher the risk of plaque rupture, which can aggravate the degree of vascular stenosis or lead to occlusion.

\subsection{Neovascularization in the plaque}

Contrast-enhanced ultrasound and superb microvascular imaging can show the location and distribution of neovascularization in plaques, which can 
promote the development of atherosclerotic lesions and even induce plaque hemorrhage and rupture, which are important factors in plaque vulnerability [7].

\section{Characteristics and Standard Description}

\section{of Vulnerable Plaques}

Expert opinion: Ultrasound can evaluate the vulnerability of a plaque, but the ultrasound should be combined with risk factors for cerebrovascular disease to ensure comprehensive analysis.

\subsection{Ultrasonic characteristics of vulnerable plaques}

(1) Plaque rupture or ulceration (discontinuous fibrous cap, pulsation with the heart beat, or a "volcanic crater" sign); (2) large lipid core or hemorrhage in the plaque (hypoechoic or echolucent characteristics) $[7,8]$; (3) contrast-enhanced ultrasound or superb microvascular imaging show that the dynamic moving hyperechoic light points extend from the adventitia to the inside of the plaque, indicating neovascularization in the plaque [9].

\subsection{Standard ultrasound report}

The standard ultrasound report of vulnerable plaques should include: (1) plaque hemorrhage, (2) thrombosis secondary to plaque rupture, (3) ulcerative plaque, and (4) neovascularization in the plaque (ultrasound contrast and/or superb microvascular imaging).

\section{Differentiating Thrombosis Secondary to Plaque Rupture}

Expert opinion: Thrombosis secondary to plaque rupture is an important mechanism in cerebrovascular events [10]. Using ultrasound examination, it is difficult to accurately identify thrombi secondary to plaque rupture. A typical thrombus formation secondary to plaque rupture has the following characteristics [11, 12]: (1) the plaque surface presents homogeneous hypoecho; (2) the unstable thrombus may show poor wall attachment or float with blood flow; (3) plaque rupture and thrombosis as a main cause of the carotid artery occlusion. Through careful examination of the pathological structure in the lumen of patients with carotid artery occlusion, clinicians define the location, outline, and fibrous cap of the potentially responsible plaque. If a vulnerable plaque of the carotid artery is found to have a discontinuous fibrous cap and hypoecho attachment on the surface, it is identified as thrombosis secondary to plaque rupture; (4) short-term follow-up has shown that thrombosis secondary to plaque rupture can also be diagnosed based on plaque volume reduction.

\section{The Value of PSV Ratio for Assessing}

\section{Carotid Artery Stenosis}

Expert opinion: The quantification of carotid artery stenosis degree is mainly based on PSV and Doppler spectrum analysis. When the presence of hypertension, hypotension, cardiac insufficiency, anemia, hyperthyroidism, and other diseases affects the measurement of arterial flow velocity, PSV ratio is more accurate than PSV or EDV to assess arterial stenosis [13]. The PSV ratio is measured between the stenosis and the proximal segment of the stenosis (PSVst/ PSVpro; Rpsv1), as well as between the stenosis and the distal segment of the stenosis (PSVst/PSVdis; Rpsv2). The PSV ratio more accurately reflects segmental hemodynamic changes caused by different degrees of artery stenosis. Taking the patient's own physiological regulation state as a reference, it mitigates individual differences in PSV, as well as the effect of PSV on the degree of stenosis in the carotid artery.

\section{Ultrasound Diagnostic Criteria for Carotid} Artery Stenosis

Expert opinion: The ultrasound evaluation criteria for carotid artery stenosis published by the North American Radiological Conference in 2003 are internationally accepted diagnostic criteria for the degree of carotid artery stenosis [13] (Table 1). In 2006, Hua et al. [14] used angiography as a reference standard to determine the diagnostic criteria of both single-parameter and multi-parameter evaluation of different degrees of carotid stenosis in a large sample (416 patients, 832 vessels). They found that the overall diagnostic accuracy reached $90 \%$ (Table 2). Hua et al. $[15,16]$ published the diagnostic criteria for stenosis of the origin of the vertebral artery and of the subclavian artery in 2009 and 2011, respectively (Table 3,4). Hemodynamic parameters are important parameters in the classification of stenosis degree, which should be comprehensively evaluated based on the PSV, EDV, and PSV ratio of the stenotic artery $[5,17]$.

\section{Differentiation of Moderate and Severe \\ Carotid Stenosis}

Expert opinion: Severe carotid stenosis should be comprehensively identified according to standard vessel diameter and hemodynamic parameters.

\subsection{Differentiation of moderate and severe internal carotid stenosis}

Identification points: the stenosis rate can be calculated based on (1) the residual and original inner 
diameter of the stenosis segment (European Carotid Surgery Trial), (2) the PSV and EDV measurement results of the stenosis segment, (3) the PSV ratio between the internal carotid artery stenosis and the common carotid artery (PSVICA/PSVCCA), (4) the PSV ratio between the stenosis segment of the internal carotid artery and the distal segment of stenosis (PSVst/PSVdis), and (5) changes in the spectral characteristics of the poststenotic segment.

Table 1 Diagnostic criteria of blood flow parameters for internal carotid artery stenosis (2003, North American Radiology Annual Conference)

\begin{tabular}{lccc}
\hline Stenosis degree & PSV, cm $/ \mathrm{s}$ & EDV, cm/s & PSVICA/PSVCCA \\
\hline Normal and $<50 \%$ & $<125$ & $<40$ & $<2.0$ \\
$50 \% \sim 69 \%$ & $125 \sim<230$ & $40 \sim<100$ & $2.0 \sim<4.0$ \\
$70 \% \sim 99 \%$ & $\geqslant 230$ & $\geqslant 100$ & $\geqslant 4.0$ \\
Occlusion & No blood flow signal & No blood flow signal & No blood flow signal \\
\hline
\end{tabular}

PSV, peak systolic velocity; EDV, end-diastolic velocity; ICA, internal carotid artery; CCA, common carotid artery.

Table 2 Differentiation of moderate from severe carotid stenosis (2006, Xuanwu Hospital, Capital Medical University)

\begin{tabular}{lccc}
\hline Stenosis degree & PSV, cm/s & EDV, cm/s & PSVst/PSVdis \\
\hline$<50 \%$ & $<155$ & $<60$ & $<2.0$ \\
$50 \% \sim 69 \%$ & $155 \sim<220$ & $60 \sim<100$ & $2.0 \sim<3.5$ \\
$70 \% \sim 99 \%$ & $\geqslant 220$ & $\geqslant 100$ & $\geqslant 3.5$ \\
Occlusion & No blood flow signal & No blood flow signal & No blood flow signal \\
\hline
\end{tabular}

PSV, peak systolic velocity; EDV, end-diastolic velocity; st, stenosis; dis, distal segment.

Table 3 Diagnostic criteria of blood flow parameters for the vertebral artery stenosis (2009, Xuanwu Hospital, Capital Medical University)

\begin{tabular}{lccc}
\hline Stenosis degree & PSV, cm/s & EDV, cm/s & PSVOR/PSVIV \\
\hline$<50 \%$ & $85 \sim<140$ & $27 \sim<35$ & $3.3 \sim<2.1$ \\
$50 \% \sim 69 \%$ & $140 \sim<220$ & $2.1 \sim<4.0$ & 250 \\
$70 \% \sim 99 \%$ & $\geqslant 220$ & No blood flow signal & No blood flow signal \\
\hline Occlusion & No blood flow signal & 4.0 \\
\hline
\end{tabular}

PSV, peak systolic velocity; EDV, end-diastolic velocity; OR, origin segment (V1 segment); IV, intervertebral segment (V2 segment).

Table 4 Diagnostic criteria for subclavian artery stenosis (2011, Xuanwu Hospital, Capital Medical University)

\begin{tabular}{lcccl}
\hline Stenosis degree & PSV, $\mathrm{cm} / \mathrm{s}$ & EDV, $\mathrm{cm} / \mathrm{s}$ & PSVOR/PSVIV & \multicolumn{1}{c}{ Vertebral artery spectrum } \\
\hline$<50 \%$ & - & - & - & No change \\
$50 \% \sim 69 \%$ & - & - & - & Notch (partial reversal) latent (partial steal) \\
$70 \% \sim 99 \%$ & $\geqslant 343$ & $\geqslant 60$ & $\geqslant 4.0$ & Partial reversal (partial steal) \\
Occlusion & No blood flow signal & No blood flow signal & No blood flow signal & Complete reversal (complete steal) \\
\hline
\end{tabular}

PSV, peak systolic velocity; EDV, end-diastolic velocity; OR, origin segment (V1 segment); IV, intervertebral segment (V2 segment); “-_" means no data.

\subsection{Differentiation of moderate and severe carotid stenosis}

In addition to the relevant criteria in Table 2, moderate and severe carotid stenosis can be identified based on (1) Spectrum morphology and resistance index (RI). In severe stenosis, the RI of the proximal segment of stenosis is increased, the flow spectrum shows highresistance waveform changes, the RI of the distal segment of stenosis is significantly/or relatively decreased, and the flow spectrum shows peak-extending low-resistance waveform changes. In moderate stenosis, there are no such characteristic flow spectrum morphology or RI changes; (2) Intracranial arterial hemodynamic changes. When the extracranial carotid artery is severely stenosed, transcranial Doppler ultrasound and/or transcranial colorcoded Doppler ultrasound detect changes in the lowresistance waveform. The spectrum of the M1 segment of the affected middle cerebral artery is prolonged at its peak, as is the spectrum of the A1 segment of the affected anterior cerebral artery (ACA). The flow velocity is relatively slow and the pulsatility index (PI) is either decreased or shows a side-to-side difference of more than 0.1 ; (3) The characteristics of collateral circulation opening. Transcranial Doppler or transcranial 
color-coded Doppler ultrasonography show the anterior communicating artery, posterior communicating artery, and ophthalmic artery collateral pathways. The opening of the collateral pathway is an important diagnostic basis for differentiating moderate from severe carotid stenosis [18].

\section{Assessment of Carotid Stenosis Degree} and the Accuracy of Diameter Reduction and

\section{Area Reduction}

Expert opinion: Assessment of the carotid stenosis degree is a comprehensive process involving diameter reduction, area reduction, and hemodynamic parameters (see above). Among these indicators, the quantitative diagnosis of localized segmental stenosis is mainly based on hemodynamic parameters, but when long segment stenosis or extremely severe stenosis lesions combined with elevation of PSV cannot meet the evaluation criteria, diagnosis should be carried out by combining the diameter reduction and the area reduction.

There are two more classical methods for calculating the percent of stenosis in the carotid artery. One is the North American Symptomatic Carotid Endarterectomy Trial (NASCET) and the other is the European Carotid Surgery Trial (ECST). According to the NASCET guidelines, the luminal diameter $(R)$ is measured proximally at the point of maximal stenosis and distally where the ICA lumen becomes normal (D); the resultant percentage stenosis is expressed as $1-\mathrm{R} / \mathrm{D} \times 100 \%$. The ECST method measures the original inner diameter (L) and residual inner diameter $(\mathrm{R})$ of the stenosis, with the following formula: $1-(\mathrm{R} / \mathrm{L}) \times 100 \%$. As a result, the calculated results of this method are higher than those of the NASCET standard. Any report of ultrasound results describing the degree of stenosis in percentage must clarify the type of radiological grading referred to: NASCET or ECST. Figure 4 shows the area reduction as follows: [1-(minimum lumen cross-sectional area/ original lumen cross-sectional area at stenosis) $] \times 100 \%$. The $50 \%$ diameter reduction corresponds to $75 \%$ of the area reduction, while assessment of the area reduction often leads to overvaluing of the stenosis severity. For this reason, the area reduction is often used as a reference only when judging the degree of stenosis. For symmetrical vessel stenosis, ultrasonic measurement of the diameter stenosis reduction is more accurate. For asymmetrical stenosis, the maximum area reduction of the blood vessel cross-section should be measured; it should then be combined with the flow velocity and spectral change characteristics of the vascular stenosis, as well as with the poststenotic segment, to comprehensively judge the degree of carotid artery stenosis.

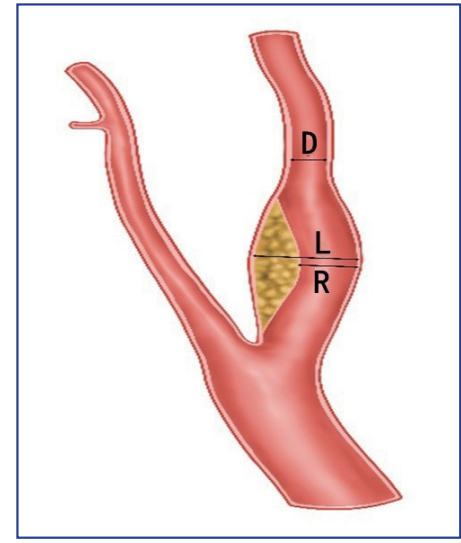

Figure 4 Standard carotid stenosis measurement diagram of the North American Symptomatic Carotid Endarterectomy Trial and European Carotid Surgery Trial. D: diameter of the internal carotid artery; L: original inner diameter at the stenosis; R: residual diameter of stenosis lumen.

\section{Judging the Degree of Stenosis of Calcified} Carotid Atherosclerotic Plaques with Acoustic Shadow

Expert opinions: The occlusion of calcified carotid plaques makes it more difficult to observe the characteristics of the deep vascular structure. In B-mode, occlusion may affect the accuracy of residual inner diameter measurement, of the original inner diameter measurement, or of the determination of vascular stenosis degree [19]. In such cases, clinicians should reduce the effect of the calcified plaque's acoustic shadow on the observation of vessel wall structural characteristics by adjusting the direction of the ultrasonic acoustic beam.

When a calcified plaque is encountered, the location and involvement of plaque on the vascular wall should be determined based on continuous cross-sectional scanning. The acoustic beam direction should then be adjusted to avoid shadow occlusion of the calcified plaque. When the calcified plaque is approximately circular and satisfactory two-dimensional imaging or CDFI images cannot be obtained by adjusting the direction of the acoustic beam, Power Doppler imaging mode, or simply pulsed wave Doppler, can be used directly to measure both the flow velocity parameters (PSV, EDV, PSV ratio) of the stenosis section, the proximal and distal segments of the stenosis. To judge the degree of stenosis, especially focusing on the change in flow velocity and spectrum characteristics of the internal carotid artery in the distal segment of stenosis and the opening of the intracranial and extracranial collateral pathways, the degree of vascular stenosis must be comprehensively judged to improve the accuracy of diagnosis.

\section{Assessment of Carotid Artery Tortuous}

Expert opinions: Carotid artery tortuosity describes a 
change in morphological characteristics caused by blood vessel prolongation. It is more common in the proximal internal carotid artery, cervical segment of the vertebral artery, and common carotid artery [20,21], and it can occur on one side or both sides. The incidence of carotid artery tortuosity is $18 \%-34 \%$, and its characteristic changes include coiling, looping, and twisting. Coiling means that the artery tortuosity is C-shaped or S-shaped (bending angle $\leqslant 90^{\circ}$, bending twice), loop-shaped refers to a $360^{\circ}$ rotation in the artery, while twisting refers to an artery tortuous angle $\geqslant 90^{\circ}$ (Fig. 5).

The mechanism of carotid artery tortuosity is still unclear, although it may be related to congenital abnormalities in the development of the arterial wall middle layer muscle fiber, atherosclerosis, hypertension, or advanced age. Congenital developmental factors are more common in adolescents, while elderly patients show agerelated degenerative changes, decreased arterial elasticity, atherosclerosis, shortened neck distance, elevated aortic arch, and other factors. It remains controversial whether carotid artery tortuosity causes clinically significant hemodynamic changes. In general, carotid artery tortuosity does not affect cerebral blood flow or cause ischemic stroke [22, 23]. Severe carotid artery tortuosity leads to hemodynamic changes and secondary clinical manifestations related to cerebral ischemia [24].

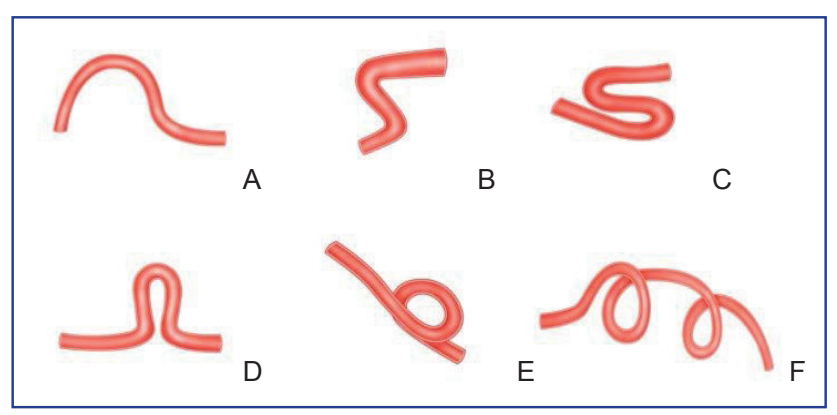

Figure 5 Ultrasound examination of internal carotid artery tortuosity. (A): C-shaped; (B): S-shaped with a bending angle of nearly $90^{\circ}$; (C): S-shaped with bending angle $\leqslant 90^{\circ}$ and bending twice; (D): loop bending; (E): loop bending of $360^{\circ}$; (F): twice consecutive loop bending of $360^{\circ}$.

Carotid artery tortuosity should be described in the ultrasound report. Generally, it does not need to be diagnosed in the conclusion. However, markedly tortuous, pulsatile masses in the neck should be described in the ultrasound report and mentioned in the conclusion, as should such masses that affect distal blood perfusion. In patients with carotid artery tortuosity and severe stenosis who require treatment using carotid endarterectomy or carotid artery stenting, the location, degree, cumulative length, distance from stenosis, and anatomical relationships of the vascular tortuosity should be detailed and mentioned in the conclusion. Such findings are of great clinical significance with regards to choosing the best treatment plan and reducing the difficulty and risk of treatment [25].

\section{Assessment of the Degree of Stenosis with}

\section{Carotid Artery Tortuous}

Expert opinion: Tortuosity in the carotid artery may cause a change in flow velocity and vascular stenosis. In the case of the latter, clinicians must be careful to distinguish it from stenosis caused by atherosclerotic lesions. When there is no stenosis in a tortuous carotid artery, its inner wall is smooth, there is no obvious abnormal echo in the lumen, and the blood flow is well filled. Furthermore, tracking and scanning along the artery shows the location, length, and morphological characteristics of the carotid artery tortuosity. CDFI can be used to observe segmental changes in blood flow direction caused by arterial tortuosity. To comprehensively judge the degree of arterial stenosis in patients with both carotid artery tortuosity and stenosis, clinicians must carefully observe whether there is an atherosclerotic lesion in the stenosis segment, narrowing of the inner diameter of the vessel, or thinning of the blood flow tract displayed by CDFI, as well as whether the patient shows increased segmental velocity accompanied by spectrum broadening, spectrum window filling, and changes in PSV, EDV, PSV ratio, and spectrum shape [13] (see "12 Ultrasound diagnostic criteria for carotid artery stenosis").

\section{Factors Influencing the Accuracy of Carotid Stenosis}

Expert opinion: The degree of carotid artery stenosis is mainly evaluated based on structural changes in the affected vessels and in hemodynamic parameters. Therefore, any factor that affects the carotid hemodynamic parameters can affect the accuracy of stenosis degree assessment, including patient factors, vascular structure factors, and operator factors [1].

\subsection{Systemic factors}

High or low blood pressure, cardiac insufficiency, hyperthyroidism, anemia, and arrhythmia (atrial fibrillation) can affect the accuracy of the spectrum characteristics and hemodynamic parameters (PSV, EDV, PSV ratio) of stenosis and poststenotic segment. Clinicians should pay attention to how collateral pathway of intracranial and extracranial arteries affects PSV and EDV.

\subsection{Vascular structure factors}

Factors such as curved or tortuous vessels, severe stenosis, or occlusive lesions in the contralateral 
carotid artery (collateral blood flow in the anterior communicating artery) may cause overestimation of the degree of stenosis [26], while tandem segmental stenosis may be underestimated. The velocity measurements in the stenosis of the echolucent plaque were relatively higher than those of echogenic plaques [27].

\subsection{Operator factors}

Sampling location: The location and accuracy of velocity measurement in the stenosis and poststenotic segment directly affect the determination of stenosis degree. Flow velocity in the poststenotic segment should be measured in the vascular lumen 4-6 cm from poststenotic segment without turbulence or eddy. The Doppler angle should be $\leqslant 60^{\circ}$ and consistent with the direction of the flow beam.

\section{Determination of Long-segment Artery}

\section{Stenosis Degree}

Expert opinion: Arterial vascular lesions with long-segment and multi-segment stenosis, as well as a stenosis rate $\geqslant 95 \%$, may show a relative decrease in flow velocity, which can be mistaken for normal (false negative). There is a difference between the hemodynamic changes caused by long-segment arterial stenosis and those caused by localized arterial stenosis [27]. According to Poiseuille's law, when fluid is in laminar flow in a horizontal pipe, the flow volume $Q$, the pressure difference between the two ends of the lumen $(\Delta \mathrm{p})$, the radius of lumen (r), blood vessel length (L), and the viscosity coefficient of the fluid $(\eta)$, have the following relationship: $\mathrm{Q}=\pi \times \mathrm{r} 4 \times \Delta \mathrm{p} /(8 \eta \mathrm{L})$ [6]. If the length of the stenosis increases, the flow volume and the flow velocity in the lumen decrease accordingly.

Therefore, for long-segment carotid artery stenosis, to reduce the difficulty of measuring flow velocity after stenosis and improve accuracy, clinicians must make a comprehensive judgment based on the characteristics of the diameter stenosis rate, the area stenosis rate of the affected vessel, the flow velocity, and the spectral changes in the stenosis and poststenotic segment.

\section{Differentiation and Instrumental Adjustment} of Complete Carotid Artery Occlusion and Subtotal Carotid Artery Occlusion

Expert opinion: Ultrasound examination should refine the degree and type of lesions in patients with internal carotid artery occlusion.

\subsection{Internal carotid artery occlusion}

Internal carotid artery occlusion can be divided into complete and subtotal types. Complete occlusion refers to abnormal echo filling in the internal carotid artery in the extracranial segment. In the CDFI mode, no blood flow signal is detected from the proximal segment to the distal segment. Subtotal occlusion refers to abnormal echo filling in the lumen of the internal carotid artery extracranial segment (4-6 cm within the visible range of ultrasound). Under CDFI or power Doppler imaging, blood flow imaging in the affected lumen shows "thin line sign," and the Doppler spectrum shows a change in the characteristics of a single peak during the systolic period, low flow velocity with high resistance, or low velocity with low resistance [5].

\subsection{Suggestions to improve the diagnostic accuracy of occlusive lesions by adjusting the instruments}

In cases of suspected internal carotid artery occlusion, clinicians should distinguish complete occlusion from subtotal occlusion, and the accuracy of lesion diagnosis can be improved by adjusting the relevant parameters detected by the instrument [27], as follows: (1) color flow imaging filtering should be adjusted to low filtering; (2) color flow velocity scale should be reduced by reducing the pulse repetition frequency, this should improve the sensitivity of low-velocity blood flow detection; (3) persistence should be increased to improve the sensitivity of low-velocity blood flow imaging; (4) the power Doppler imaging mode should be used to improve the sensitivity of low-velocity blood flow signal imaging; (5) the direction of the probe acoustic beam should be adjusted to reduce the examination angle between the acoustic beam and the blood flow; (6) if necessary, the contrast-enhanced ultrasound should be used to improve the accuracy with which occlusion and subtotal occlusion of the internal carotid artery are diagnosed.

\section{Expert Group (in no particular order)}

Yang Hua, $M D^{a}$, Yingqi Xing, $M D^{a}$, Lingyun Jia, $M D^{a}$, Yumei Liu, $M D^{a}$, Xi Liu, MD ${ }^{b}$, Shumin Wang, $M D^{c}$, Xuan Meng, MD ${ }^{d}$, Feng Zhang, $M D^{e}$, Kun Liu, $M D^{f}$, Binbin Song, $M D^{g}$, Xiangli Xu, $M D^{h}$, Yanyan Jiang, $M D^{i}$, Tao Peng, $M D^{j}$, Yajie Li, MD ${ }^{k}$, Chunmei Zhang, $M D^{l}$, Xiaozhi Zheng, $M D^{m}$, Yan Li, $M D^{n}$, Tianan Jiang, $M D^{o}$, Chaoyang Wen, $M D^{p}$

\section{Secretary Group (in no particular order)}

\footnotetext{
Xiaojie Tian, $M D^{a}$, Xiujing Xie, $M D^{\circ}$, Min Xu, $M D^{o}$

${ }^{a}$ Xuanwu Hospital, Capital Medical University; ${ }^{b}$ Air Force Medical Center; ${ }^{c}$ Peking University Third Hospital; ${ }^{d}$ Lanzhou University Second Hospital; ${ }^{e}$ The Sixth Affiliated Hospital of Sun Yat-sen University; ${ }^{f}$ The Third People's Hospital of Hubei Province; ${ }^{g}$ Luoyang Central Hospital Affiliated to Zhengzhou University; ${ }^{h}$ The Second Hospital of Harbin; ${ }^{i} 900$ Hospital of the Jiont Logistics
} 
team; ${ }^{j}$ The Second People's Hospital of Liaocheng; ${ }^{k}$ The Centre Hospital of Jilin City; ' The First Affiliated Hospital of Harbin Medical University; ${ }^{m}$ Yangpu Hospital, Tongji University School of Medicine; ${ }^{n}$ Beijing Luhe Hospital, Capital Medical University; ${ }^{\circ}$ The First Affiliated Hospital, College of Medicine, Zhejiang University; ${ }^{p}$ Peking University International Hospital

\section{Declaration}

This Expert Consensus was organized by The Professional Committee of Vascular Ultrasound of Stroke Prevention and Treatment Expert, Committee of the National Health Commission; The Professional Committee of Superficial Organ and Peripheral Vascular Ultrasound of the Chinese Medical Ultrasound Engineering; The Professional Committee of Craniocerebral and Cervical Vascular Ultrasound of the Chinese Medical Ultrasound Engineering.

The contents of this article in Chinese was peerreviewed and published by the Chinese Journal of Cerebrovascular Diseases for 2020 17(6):346-353. This English version of the consensus was adapted from the Chinese one with permission. The link of Chinese vision of the article is as follows: www.cjevd.cn.

\section{Conflict of Interest}

The authors have no conflict of interest to declare.

\section{References}

[1] Yang Hua. Practical Ultrasound Diagnosis of Carotid Artery and Cerebral Vessels. Beijing: Science Press; 2002. p149-54.

[2] John S Pellerito, Joseph F Polak. Introduction to Vascular Ultrasonography (6th edition). Philadelphia: Saunders; 2012. p2526.

[3] Stroke Prevention and Treatment Expert Committee of the National Health Commission of China. Guidelines for vascular ultrasound examination of stroke in China. Chin J Med Ultrasound (Electronic edition) 2015; 12: 599-610.

[4] Fishbein GA, Fishbein MC. Arteriosclerosis: rethinking the current classification. Arch Pathol Lab Med 2009; 133: 1309-16.

[5] László Csiba, Claudio Baracchini. Manual of Neurosonology. Cambridge: Cambridge University Press; 2016. p58-59.

[6] William J. Zwiebel. Introduction to Vascular Ultrasonography (4th Edition). Amsterdam: Elsevier; 2000. p155-169.

[7] Gonçalves I, den Ruijter H, Nahrendorf M, Pasterkamp G. Detecting the vulnerable plaque in patients. J Intern Med 2015; 278: 520-30.

[8] Picano E, Paterni M. Ultrasound tissue characterization of vulnerable atherosclerotic plaque. Int J Mol Sci 2015; 16: 10121-33.

[9] Amamoto T, Sakata N, Ogata T, Shimada H, Inoue T. Intra-plaque vessels on contrast-enhanced ultrasound sonography predict carotid plaque histology. Cerebrovasc Dis 2018; 46: 265-9.

[10] Spagnoli LG, Mauriello A, Sangiorgi G, Fratoni S, Bonanno E, Schwartz RS, et al. Extracranial thrombotically active carotid plaque as a risk factor for ischemic stroke. JAMA 2004; 292: 1845-52.

[11] Weintraub HS. Identifying the vulnerable patient with rupture-prone plaque. Am J Cardiol 2008; 101: 3F-10F.

[12] Ohyama H, Mizushige K, Takahashi T, Hosomi N, Kohno M. Plaque rupture on the carotid artery observed by Doppler ultrasonography--a case report. Angiology 2001; 52: 867-9.

[13] Grant EG, Benson CB, Moneta GL, Alexandrov AV, Baker JD, Bluth EI, et al. Carotid artery stenosis: gray scale and Doppler US diagnosis--society of radiologists in ultrasound consensus conference. Radiology 2003; 229: 340-6.

[14] Hua Y, Liu BB, Ling C, Duan C, Liu Q, Miao Z, et al. Accurate assessment of the diagnosis between $50-69 \%$ and $70-99 \%$ carotid stenoses with ultrasonography. Chin J Cerebrovasc Dis 2006; 3: 2118.

[15] Hua Y, Meng XF, Jia LY, Ling C, Miao ZR, Ling F, et al. Color Doppler imaging evaluation of proximal vertebral artery stenosis. AJR Am J Roentgenol 2009; 193: 1434-8.

[16] Hua Y, Jia LY, Li L, Ling C, Miao ZR, Jiao LQ. Evaluation of severe subclavian artery stenosis by color Doppler flow imaging. Ultrasound Med Biol 2011; 37: 358-63.

[17] Dharmasaroja PA, Uransilp N, Watcharakorn A, Piyabhan P. Accuracy of carotid Duplex criteria in diagnosis of significant carotid stenosis in Asian patients. J Stroke Cerebrovasc Dis 2018; 27: 77882.

[18] Hua Y, Ling C, Duan C, Zhang L. Evaluation of accuracy for combining color duplex imaging and transcranial Doppler ultrasound in diagnosing severe stenosis or occlusion of internal carotid artery. Chin J Ultrasonogr 2000; 9: 413-5.

[19] Mohebali J, Patel VI, Romero JM, Hannon KM, Jaff MR, Cambria $\mathrm{RP}$, et al. Acoustic shadowing impairs accurate characterization of stenosis in carotid ultrasound examinations. J Vasc Surg 2015; 62: 1236-44.

[20] Matula C, Trattnig S, Tschabitscher M, Day JD, Koos WT. The course of the prevertebral segment of the vertebral artery: anatomy and clinical significance. Surg Neurol 1997; 48: 125-31.

[21] Nagata T, Masumoto K, Hayashi Y, Watanabe Y, Kato Y, Katou F. Three-dimensional computed tomographic analysis of variations of the carotid artery. J Craniomaxillofac Surg 2016; 44: 734-42.

[22] Beigelman R, Izaguirre A, Robles M, Grana D, Ambrosio G, Milei J. Kinking of carotidarteries is not a mechanism of cerebral ischemia: a functional evaluation by Doppler echography. Int Angiol 2011; 30: $342-8$.

[23] Togay-Işikay C, Kim J, Betterman K, Andrews C, Meads D, Tesh $\mathrm{P}$, et al. Carotid artery tortuosity, kinking, coiling: stroke risk factor, marker, or curiosity? Acta Neurol Belg 2005; 105: 68-72.

[24] Cvetko E. Concurrence of bilateral kinking of the extracranial part of the internal carotid artery with coiling and tortuosity of the external carotid artery-a case report. Rom Morphol Embryol 2014; 55: 4335.

[25] Illuminati G, Ricco JB, Caliò FG, D'Urso A, Ceccanei G, Vietri F. Results in a consecutive series of 83 surgical corrections of symptomatic stenotic kinking of the internal carotid artery. Surgery 2008; 143:134-9.

[26] Xia MY, Hua Y, Jia LY, Zhou YH. Study on overestimation of blood flow velocity after bilateral internal carotid artery stenosis with color Doppler ultrasound. Chin J Cerebrovas Dis 2011; 8: 570-5.

[27] Romero JM, Lev MH, Chan ST, Connelly MM, Curiel RC, Jackson $\mathrm{AE}$, et al. US of neurovascular occlusive disease: interpretive pearls and pitfalls. Radiographics 2002; 22: 1165-76. 